\title{
Auroral classification ergonomics and the implications for machine learning
}

\author{
Derek McKay ${ }^{1}$ and Andreas Kvammen ${ }^{2}$ \\ ${ }^{1}$ NORCE Norwegian Research Centre AS, Troms $\varnothing$, Norway \\ ${ }^{2}$ Department of Physics and Technology, UiT - The Arctic University of Norway, Troms $\varnothing$, Norway
}

Correspondence: Derek McKay (demc@norceresearch.no)

Received: 6 December 2019 - Discussion started: 28 January 2020

Revised: 6 June 2020 - Accepted: 16 June 2020 - Published: 9 July 2020

\begin{abstract}
The machine-learning research community has focused greatly on bias in algorithms and have identified different manifestations of it. Bias in training samples is recognised as a potential source of prejudice in machine learning. It can be introduced by the human experts who define the training sets. As machine-learning techniques are being applied to auroral classification, it is important to identify and address potential sources of expert-injected bias. In an ongoing study, 13947 auroral images were manually classified with significant differences between classifications. This large dataset allowed for the identification of some of these biases, especially those originating as a result of the ergonomics of the classification process. These findings are presented in this paper to serve as a checklist for improving training data integrity, not just for expert classifications, but also for crowd-sourced, citizen science projects. As the application of machine-learning techniques to auroral research is relatively new, it is important that biases are identified and addressed before they become endemic in the corpus of training data.
\end{abstract}

\section{Introduction}

Each year, the all-sky cameras in the Arctic and Antarctic regions collect several million images of the sky. These contain a plethora of atmospheric and astronomical phenomena including, of particular interest to the authors, manifestations of the aurorae. Auroral emissions are excited when charged particles from the magnetosphere enter the ionosphere and collide with atoms, molecules and ions in the ionosphere. The flux of energetic charged particles entering the ionosphere is dependent on solar wind as well as magnetospheric and ionospheric conditions. Different conditions will result in different auroral features; thus, the auroral sky acts as a window into the otherwise obscure solar windmagnetosphere-ionosphere environment. Having computer algorithms to pick out interesting features, or features that have potential for scientifically interesting phenomena, is helpful for scientists in auroral research.

Examination of what can be done using machine learning for such interests has been pursued, and there are other groups doing the same. Ideally, autonomous software would take a set of images and identify those which contain aurorae and, in these cases, which morphological types are present (break-up, arcs, discrete, patchy etc.).

Yet, although algorithms for the identification of visual features have made remarkable progress, these tend to be "exceptionally data-hungry". It is well-established that it is expensive and tedious to produce large, labelled training datasets, especially in cases in which expert knowledge is required (e.g. Yu et al., 2015).

Although initial attempts have been made to undertake automatic auroral classification, these have not been particularly successful (low prediction rates) or useful (high accuracy, but the categories are so broad as to not really be of significant benefit). Those programmes which have demonstrated success have focused on very specific subgroups (e.g. Yang et al., 2019).

Part of the problem with low success rates for prediction is the presence of prediction bias (e.g. Domingos, 2000). This can be attributed to various causes, such as the following. 
- Noisy training data

- An incomplete feature set

- Strong regularisation

- Algorithmic errors

- Biased training samples

In order to address these issues, a programme was undertaken to improve the reliability of machine-learning results (Kvammen et al., 2020a). In addition to using more up-to-date machine-learning methods, attention was paid to the provision of a comprehensive dataset for training the algorithms. As a part of this process, it was deemed important to remove sources of bias in the classification of the training dataset. Following some preliminary work with small samples of both greyscale and colour images, a main classification run was undertaken.

As the differences between the classifications of informed researchers were significant, the findings are presented here. It is intended that they will serve as a reference point for other endeavours in the development of machine-learning training and test sets for auroral research, but also for any other field in which machine-learning image recognition is developed from specialised sets categorised by subject experts. The paper concludes with a discussion, including references to similar work from other disciplines and suggestions for future work.

\section{Methodology}

Images from the Kiruna all-sky camera (location: $67.84^{\circ} \mathrm{N}$, $20.41^{\circ} \mathrm{E} ; 425 \mathrm{~m}$ above mean sea level; operated by the Swedish Institute for Space Physics) were used. The cam-

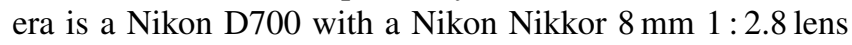
giving an almost $180^{\circ}$ field of view. The exposure time is $6 \mathrm{~s}$ taken automatically on each minute. To ease data transfer rates and processing the "quick-look summary" JPEG images $(720 \times 479$ pixels $)$ were used, rather than the fullresolution images. Approximately 300000 of these images from nine winter seasons were filtered down to a set of 13947 for human classification (by removing cloudy, moonlit and twilight images). A $3 \times 3$ median filter was applied to filter out points (stars, defective pixels, etc.), then the data were binned using a $2 \times 2$ averaging window to reduce the size of the images for neural network training. The central $128 \times 128$ pixels were then selected. This removes the horizon, where distortion is more pronounced and where light pollution and atmospheric effects are typically found. Additional information about the pre-processing and machine learning is reported in Kvammen et al. (2020a).

Two auroral physicists each classified these 13947 processed images by hand using different software implementations (one using Python, the other using MATLAB). The motivation to do this was based on knowledge of the different systems (thus making self-maintenance of the code possible) and as a way of working independently to ensure robust results.

The classification was done according to nine possible classes. The labels are listed in Table 1 along with a brief description of each label; a more thorough description of the labels and the labelling procedure is available in Kvammen et al. (2020a). These classes were the result of several iterations of planning, whereby the two experts, together with a machine-learning researcher, identified categories which would be scientifically useful, possible to discern with a reasonable algorithmic network and suitable for the sample size available. Sample images (after pre-processing) illustrating each auroral label are presented in Fig. 1.

After comparison of results, it was found that the experts only agreed on $54 \%$ of the images, with the most disagreement being on which images were suitable for training and which had an aurora with an unknown and/or complicated form. When it was agreed that the image was suitable for algorithm training, the experts agreed on $95 \%$ of the labels. By only using the images with agreeing auroral labels (i.e. bother experts independently reached the same classification) and by excluding the images with ambiguous auroral forms, unwanted features and disagreeing labels, a clean training dataset was produced at the price of excluding approximately $73 \%$ of the 13947 images in the initial dataset. The experimental results of the labelled dataset, as derived from the machine-learning study, are not considered in this paper but are presented in Kvammen et al. (2020a).

\section{Ergonomic categories}

The comparison of the classifications for both the trials and the main classification run allowed for the identification of emerging biases based on the approach each researcher took to identify the aurorae in the images. These biases are a result of the levels of comfort (physical and cognitive) that exist during a classification process, leading to the term "classification ergonomics". Those identified as part of this study are shown in Table 2 and are discussed in the subsequent sections.

\subsection{Physical comfort bias}

The classification of the aurorae in the main study was a nineclass system. Given the designations, the number keys were the obvious choice, and the classification software used these either on the main keyboard (0-8) or the numeric keypad (KP0-KP8). In the case of a mistake, it was possible to go back to the previous image, and the backspace key was used to accomplish this. This key configuration is shown in Fig. 2.

The first bias that was noted was the inconvenience of the backspace for making corrections. This required moving the 
Table 1. The set of aurora labels used for the classification run.

\begin{tabular}{lll}
\hline Label no. & Label & Description \\
\hline 0 & Auroral break-up & Bright auroral forms that cover most of the image \\
1 & Coloured aurora & The auroral emission is clearly not monochromatic green \\
2 & Auroral arcs & Auroral structures with clear east-west-aligned form \\
3 & Discrete-irregular & A combination of broken arcs, north-south-aligned arcs and vortical structures \\
4 & Patchy aurora & Aurora appears as irregular blobs or stripes on a diffuse background \\
5 & Edge aurora & Auroral emission only at the edge of the framed image \\
6 & Faint-clear & Auroral emission is not clearly visible \\
7 & Unknown or ambiguous & Aurora does not fit any of the labels above or is a mixture of several labels \\
8 & Rejected & The image is not suitable for training due to e.g. light pollution, clouds, noise \\
\hline
\end{tabular}
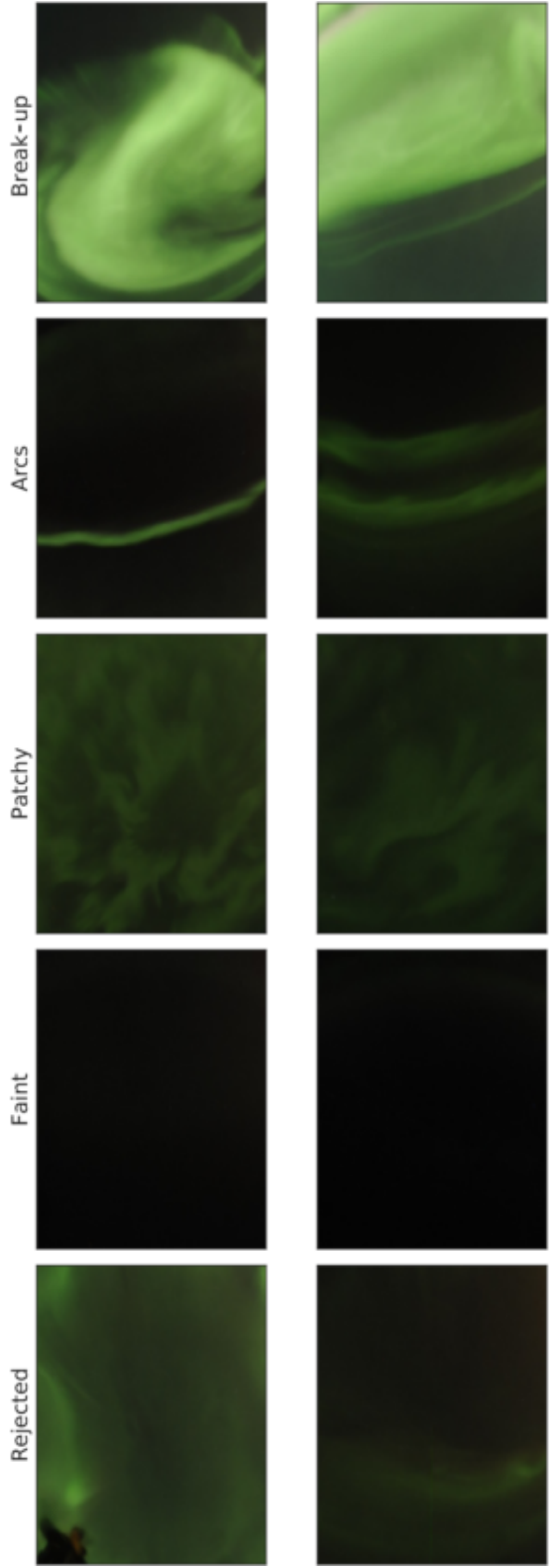
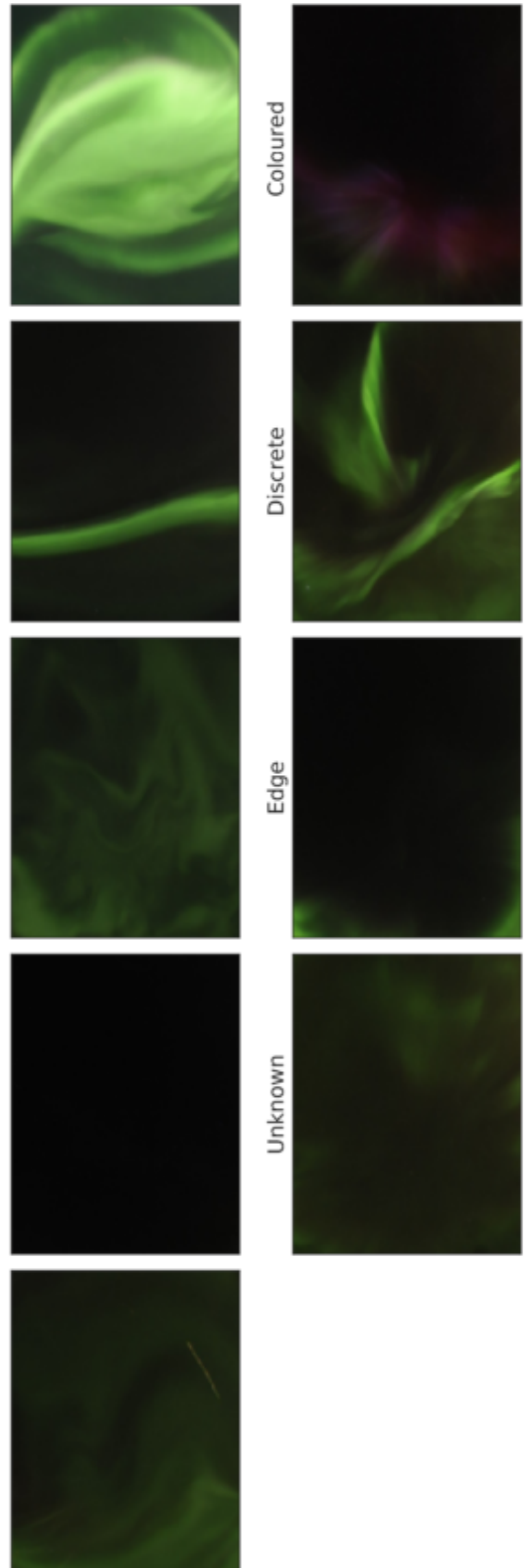
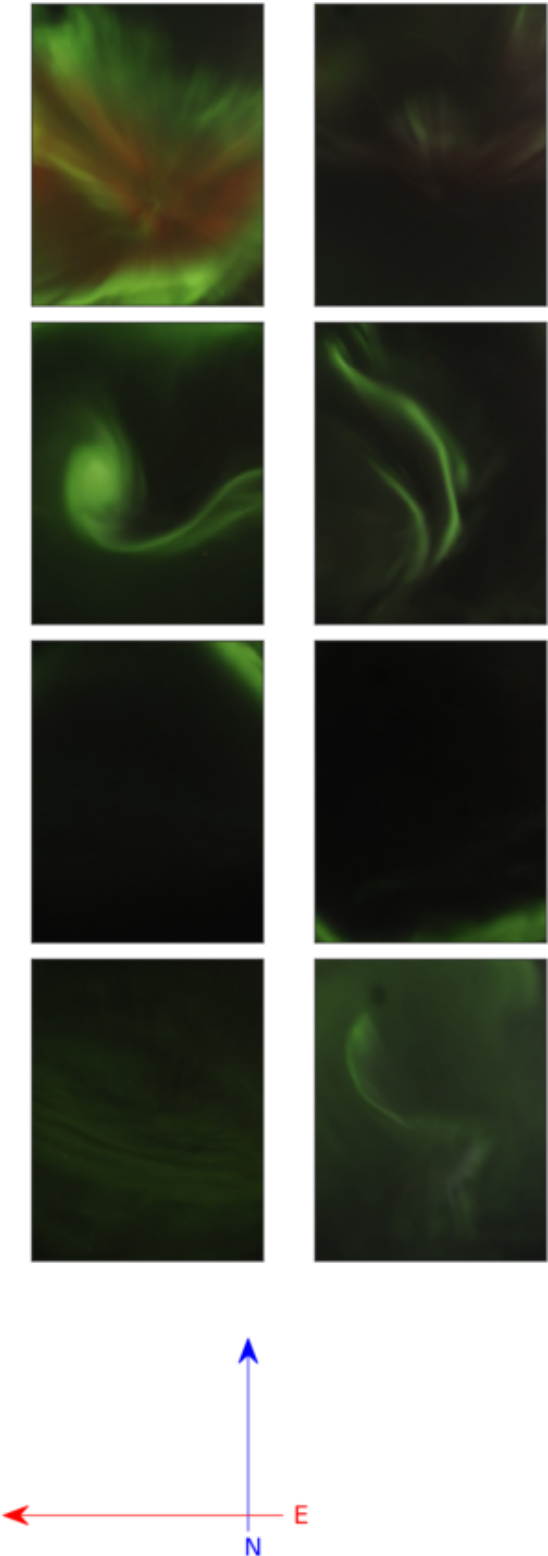

Figure 1. Sample images of each label. The direction with respect to the magnetic pole is indicated by the arrows at the bottom right. The rejected images at the bottom left are rejected due to, from left to right, a person in the bottom left corner, lidar emission and passing aircraft. 
Table 2. A set of biases that may affect user classification.

\begin{tabular}{l}
\hline Physical comfort bias \\
Data contrast bias \\
Environment contrast bias \\
Repetition bias \\
Learning bias \\
Feature bias \\
Ambiguity bias \\
Expert knowledge bias \\
\hline
\end{tabular}
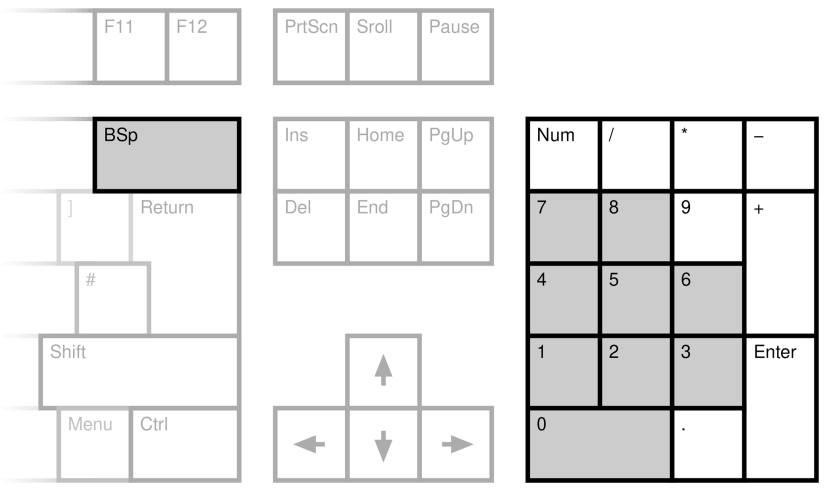

Figure 2. The original right-hand key set for classification. The grey-shaded keys were used for the classification.

right hand completely away from the rest position where the fingers hover over the KP4, KP5 and KP6 keys on the keypad. As this was awkward, there was a perceptible reluctance to make corrections. Thus, the KP-DECIMAL (to the right of the KP0 key) was used as an alias.

After several hundred classifications, discomfort was experienced, even with the keyboard rotated $10-20^{\circ}$ anticlockwise to make the keys suit the angle of the right hand. As a result, some testing was also done with more comfortable key arrangements. This resulted in a basic WASD configuration being used. WASD refers to the directional (move forward, backward, left and right) keys as used in FPS (first-person shooter) computer games.

This configuration is shown in Fig. 3, where the coloured circles show the at-rest position of the fingers (keys A, S and D), with the arrows showing easy-reach positions. The left thumb rests on the space bar. The little finger typically can reach the shift and control keys (as a modifier; in FPS games this might be e.g. run and crouch) but were not used here. The actual keys that were used for the classification are shaded in grey.

Additionally, the keyboard was rotated $10-15^{\circ}$ clockwise to match the natural angle of the left wrist and hand, as shown in Fig. 4. This was used for most of the classification work, and no discomfort was experienced.
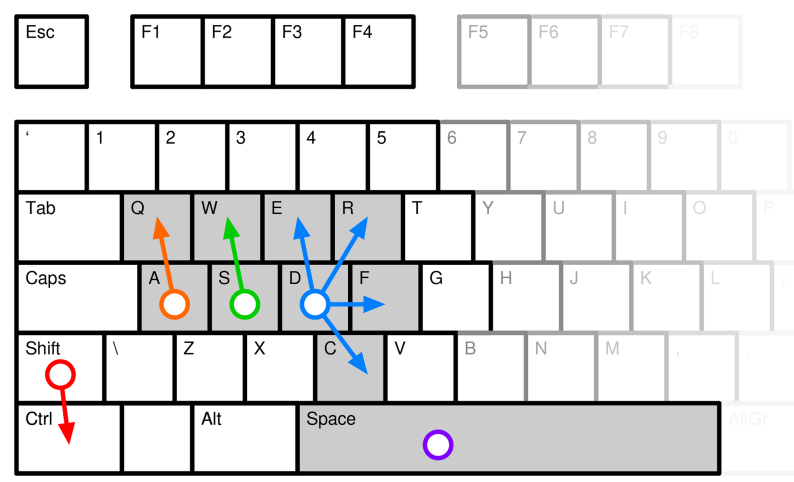

Figure 3. A left-hand key set for classification. The coloured circles show the at-rest position of the fingers, with the arrows showing easy-reach positions. The grey-shaded keys were used for the classification. See also Fig. 4.

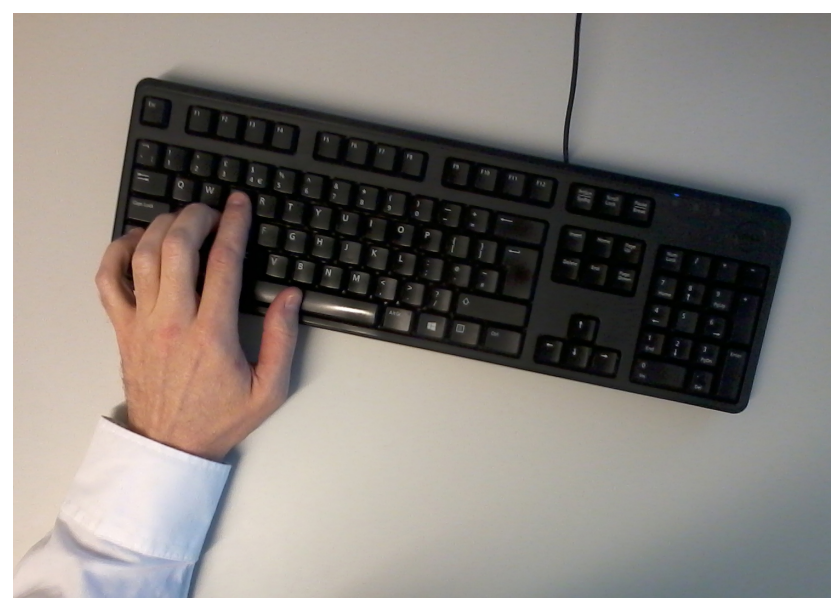

Figure 4. Hand position for the left-hand key set, with the keyboard at an angle of $10-15^{\circ}$ to minimise finger reach strain.

\subsection{Data contrast bias}

If the classifier has just seen a faint, patchy aurora, then a following faint, patchy aurora is likely to be classified the same. If the preceding image was a bright break-up, then it is more likely for the faint, patchy aurora to be classified as blank. In the initial parts of the study, attempts were made to mitigate this by normalising the image scale of all images. This was not readily achieved with colour images and thus not pursued. In the study, the classifications were done both randomly and chronologically by the two experts. Repeated, random classification would be best but was not possible within the time limitation of the project. The chronological classification allows knowledge of the substorm process to be applied to obtain a more reliable result, although this introduces anticipation of the phenomenon rather than an objective evaluation of each image on its own characteristics (Sect. 3.8). 


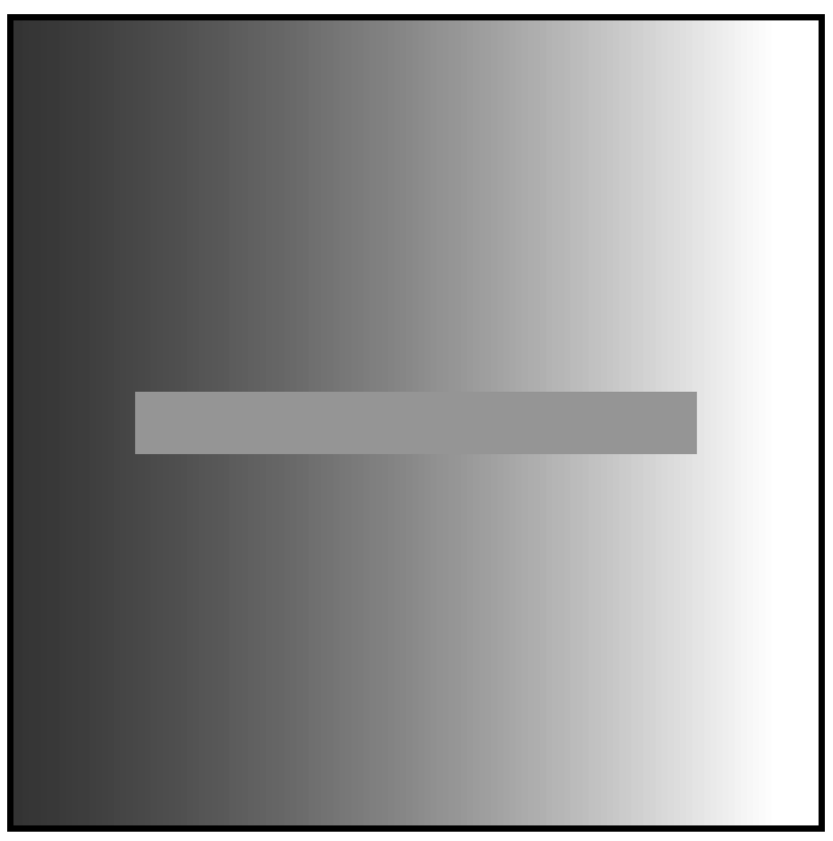

Figure 5. Example of environment contrast. The central bar may seem to be a gradient, but it is uniform in shade. Cover the surrounding (real) gradient with paper of uniform colour to demonstrate this.

\subsection{Environment contrast bias}

Humans naturally retain perceptual constancy. This allows visual features to be discerned against a noisy or changing background: a trait that is useful to all animals in a hunterprey scenario, for instance. However, this human trait of retaining perceptual constancy results in optical illusions. Colour constancy and brightness constancy will cause an illusion of colour or contrast difference when the luminosity or colour of the area surrounding an object is changed. The eye partly does this as a result of compensating for the overall lighting (change in the iris aperture), but the brain also compensates for subtle changes within the field of view. An example of this is shown in Fig. 5.

Originally, the software presented the image with a white border (the default for the plotting software). However, the contrast made it difficult to discern the difference between features which were faint but still recognisable and those which were sub-threshold for visual identification. Hence, the figure background was changed to black. This made it easier to discern the borderline cases.

The environmental conditions beyond the computer screen were also significant, with differences in the ambient lighting and room brightness being an issue. This was noted, and consistency in the arrangements for the process is likewise recommended for future studies.

\subsection{Repetition bias}

It is more comfortable to press the same button twice than to press two different buttons. Additionally, if a mistake is made, it is extra effort to go back and correct it. This "laziness" accumulates during the classification process, making long sessions problematic.

For example, if there are 10 similar images in a row, the chance of classifying number 11 in the same way is higher than if there were 10 random images first. In the study single-repetition bias was $27 \%$, rising to $40 \%$ for doublerepetition bias. It can be mitigated with randomisation (but see Sect. 3.2), whereby different experts are presented with a different random sequence of images. Discrepancy between classifications can then be investigated or the images discarded.

\subsection{Learning bias}

If there are lots of categories, the classifier may not necessarily hold all of them in mind. Thus, some "sectors" of the classification may have a higher activation energy than others. For example, when classifying hundreds of arcs and patchy aurorae and then getting a discrete case, the classifier may subconsciously think "it is not patchy, so it must be an arc", thereby inadvertently omitting the thought of a different class. This is a recency effect (whereby a new classification is biased toward the set of most recently used labels) which has been reported in the biological sciences (Culverhouse, 2007). Randomisation can be used, but re-classification can also be employed to test for variation due to learning.

\subsection{Feature bias}

The classifier is more likely to get the classification of a prominent feature correct than faint or diffuse features. This leads to a form of confusion bias; e.g. what to do with a bright discrete aurora (Class 3 ) on a background of diffuse patchy aurora (Class 4).

There is also positivity bias, whereby identification is biased by prior expectations (Culverhouse, 2007). In auroral classification, the substorm progression (development of the auroral display) makes it possible to anticipate the next image. This is partially mitigated by randomising the samples, but this can lead to contrast bias (Sect. 3.2).

\subsection{Ambiguity bias}

Ambiguity bias occurs when there is confusion as to what a particular image may be. This is exacerbated by feature bias (Sect. 3.6). However, even in cases in which there is no dominant feature, the classifier will tend to subconsciously identify some feature and latch onto it to the exclusion of other features in the image. For the main study, it was decided that ambiguous images should be rejected to make the learning environment clearer for the machine-learning algo- 
rithms - thus, such ambiguities were undesirable. What was noted was that, especially early in the study, the users would tend to try to classify the auroral image rather than reject it. When it was clear that there was no shortage of data, this tendency was reduced.

Nevertheless, it is recommended that there is a clarification of classification rules, making it clear what the user should do in a case of mixed features. If there is a precedence or priority of forms, then that should also be made very clear. Even so, ambiguities and borderline cases will remain. When data volume allows, these could be discarded.

\subsection{Expert knowledge bias}

Differences in "expert knowledge" that affect the results have also been seen. For example, although the two expert researchers involved are knowledgeable in auroral physics and its optical manifestation in general, one had done research on auroral arcs, whereas the other had not. The specialist was more picky on the arc classification (classifying $15 \%$ fewer), partially as a result of having a deeper understanding of the underlying physics but also partly in terms of having seen many more images prior to approaching the classification task. This led to a higher level of discernment on that particular category. This can be mitigated by establishing clear guidelines and "recognition cards" to assist the classification process. In cases in which there is ambiguity or disagreement, revision of the characteristics being used to do the classification can be carried out and re-classification done where necessary.

\section{Discussion}

The application of machine learning to auroral classification is an area in which only a few studies have been carried out. However, development is now progressing rapidly and it is likely that it will be applied much more and become an important part of auroral research in the future. Therefore, it is vitally important to properly address the ergonomics and biases sooner rather than later in order to avoid inadvertently introducing errors and biases early in the establishment of this new area of science.

Discrepancy between expert classifiers has been reported before. A previous auroral study had two experts that agreed on the class in about $70 \%$ of the images, and the experts chose the unknown class in almost $50 \%$ of all images (Syrjäsuo et al., 2007). However, an analysis of potential reasons for the discrepancy was not included. Similarly, a biological study found that trained personnel achieve $67 \%$ to $83 \%$ self-consistency and $43 \%$ consensus in expert taxonomic labelling tasks, with those routinely engaged in particular discriminations returning accuracies in the range of $84 \%$ to $95 \%$ (Culverhouse et al., 2003).
It is surmised that, in addition to ambiguity over the content, there is an ergonomic factor that contributes to classification bias. In any general image classifications (e.g. car vs. house or tree vs. dog), common knowledge, massive samples of people doing the training, and clear-cut distinctions between the objects make it easier (although not completely) to avoid subjective bias or even prejudice. But when the classification is being done by a small number of experts with built-in knowledge and subject background, then the training set can readily become subject to inadvertent bias. However, as a specialist field, there may be no choice. The general public may not be able to know the difference between auroral types (at least not without some training, itself subject to interpretation).

Four key human traits that affect classification performance are (a) a short-term memory limit of five to nine items, (b) boredom and fatigue, (c) recency effects whereby a new classification is biased toward the set of most recently used labels, and (d) positivity bias, wherein identification is biased by prior expectations (Culverhouse, 2007). Ambient noise, high ambient temperature, difficulty of discerning auroral features, and lack of sleep decrease performance. Additionally, attention should be paid to error analysis and associated quality metrics to weight not just algorithms, but also human-based classification according to performance (Zhu et al., 2014).

As a recommendation for future studies, classifying images in random and chronological order could be supplemented by a classification in reverse chronological order, with an examination of the results for potential hysteresis in the category selected. Specifically designed experiments could also be devised to test different biases in isolation. Ideally, these would be more generic, applying to machinevision training more generally rather than the specific aurora case presented here.

\section{Conclusions}

Ergonomics refers to the design factors intended to improve productivity by reducing the fatigue and discomfort of the user. As part of the ongoing study, the trade-off between user fatigue and scientific bias is considered. When considering the training of a classification scheme, it is important to reconcile the aspects of the task which cause scientific bias but which improve overall efficiency. Given the nature of large classification programmes, removing sources of repetitive and cognitive strain not only serve to improve the working condition of the user, but also assist in ensuring that no work environment bias is injected into training datasets that are later used for classification. The items presented in Table 2 serve as a checklist for researchers who are working in machine learning.

This is of particular use for specialist fields (such as auroral research), in which it is necessary to use a small number 
of experts to train algorithms. Consensus on any given classification is important in reducing errors in the training set, yet it is typical for experts to operate in very small teams or even alone. Addressing these issues will help future studies find a balance between the statistical effectiveness of large samples and the potential for scientific bias which may result from inappropriate ergonomic design that facilitates large sample classifications. This is particularly important for auroral research, in which the application of machine learning is relatively new, and there is much potential for misguided research on the grounds of biased input data.

Data availability. Original source images are publicly available from the Swedish Institute for Space Physics archive at http: //www2.irf.se/allsky/data.html (Brändström, 2020), and the processed image dataset and code used in this paper are available at https://doi.org/10.18710/SSA38J (Kvammen et al., 2020b). However, users are obliged to contact the Kiruna Atmospheric and Geophysical Observatory before usage of the images. The classification from these images by the authors has been submitted as a Supplement with this paper.

Supplement. The supplement related to this article is available online at: https://doi.org/10.5194/gi-9-267-2020-supplement.

Author contributions. DM and AK jointly established the classification definitions. DM and AK independently developed their classification tools and applied them to the dataset. The text and figures for this paper were produced by DM, with comments and editing by AK.

Competing interests. The authors declare that they have no conflict of interest.

Acknowledgements. The authors wish to thank Kristoffer Wickstrøm, Noora Partamies, Charlie Negri, Tuula Paavilainen and Alvin Panther for their contributions to this work. In addition, the authors would like to thank Urban Brändström and the Swedish Institute of Space Physics for providing the original auroral image data. Andreas Kvammen is supported by the Troms $\varnothing$ Research Foundation.

Financial support. Andreas Kvammen is supported by the Troms $\emptyset$ Research Foundation.
Review statement. This paper was edited by Flavia Tauro and reviewed by two anonymous referees.

\section{References}

Brändström, U.: Kiruna All-Sky Camera, Swedish Institute of Space Physics, available at: http://www2.irf.se/allsky/data.html, last access: 7 July 2020.

Culverhouse, P. F.: Human and machine factors in algae monitoring performance, Ecol. Inform., 2, 361-366, https://doi.org/10.1016/j.ecoinf.2007.07.001, 2007.

Culverhouse, P. F., Williams, R., Reguera, B., Herry, V., and González-Gil, S.: Do experts make mistakes? A comparison of human and machine indentification of dinoflagellates, Mar. Ecol.-Prog. Ser., 247, 17-25, https://doi.org/10.3354/meps247017, 2003.

Domingos, P.: A unified bias-variance decomposition, in: ICML '00: Proceedings of the Seventeenth International Conference on Machine Learning, edited by: Langley, P., Morgan Kaufmann Publishers Inc., San Francisco, CA, USA, 231-238, 2000.

Kvammen, A., Wickstrøm, K., McKay, D., and Partamies, N.: Auroral Image Classification with Deep Neural Networks, https://doi.org/10.1002/essoar.10501968.1 online first, 2020a.

Kvammen, A., Wickstrøm, K., McKay, D., and Partamies, N.: Replication Data for: Auroral Image Classification with Deep Neural Networks, DataverseNO, https://doi.org/10.18710/SSA38J, 2020b.

Syrjäsuo, M., Donovan, E., Qin, X., and Yang, Y.: Automatic classification of auroral images in substorm studies, in: 8th International Conference on Substorms (ICS8), 309-313, available at: http://aurora.phys.ucalgary.ca/donovan/pdfs/syrjaesuo_ ics8.pdf (last access: 7 July 2020), 2007.

Yang, B., Spanswick, E., Liang, J., Grono, E., and Donovan, E.: Responses of Different Types of Pulsating Aurora in Cosmic Noise Absorption, Geophys. Res. Lett., 46, 5717-5724, https://doi.org/10.1029/2019GL083289, 2019.

Yu, F., Seff, A., Zhang, Y., Song, S., Funkhouser, T., and Xiao, J.: LSUN: Construction of a Large-scale Image Dataset using Deep Learning with Humans in the Loop, available at: https://arxiv. org/pdf/1506.03365.pdf (last access: 7 July 2020), 2015.

Zhu, T., Johnson, A. E. W., Behar, J., and Clifford, G. D.: Crowd-Sourced Annotation of ECG Signals Using Contextual Information, Ann. Biomed. Eng., 42, 871-884, https://doi.org/10.1007/s10439-013-0964-6, 2014. 